\title{
An approach to uncertainty in emergency service systems via scenarios and fuzzy values
}

\author{
Jaroslav Janáček ${ }^{1, \dagger}$ and Marek Kvet ${ }^{1}$ \\ ${ }^{1}$ Faculty of Management Science and Informatics, University of Žilina, Univerzitná \\ 8215/1, 01026 Žilina, Slovakia \\ E-mail: 〈\{jaroslav.janacek, marek.kvet\}@fri.uniza.sk〉
}

\begin{abstract}
When a service system is being designed, its resistance to randomly occurring detrimental events is often assessed. Several approaches can be used to include the influence of the events in the design process. This contribution deals with two such approaches. The first approach is based on making the system resistant to a finite set of scenarios. The second approach takes input data as fuzzy values and seeks a design solution where the objective function value belongs to a fuzzy set of good objective function values at the maximal level of satisfaction. Each approach models uncertainty in a different way and we will focus on studying the impact of the used uncertainty model on the resulting minsum optimal emergency service system design, which is characterized by a deployment of a limited number of service centers in a serviced geographical region.
\end{abstract}

Keywords: location, emergency system design, scenarios, fuzzy values, min-sum

Received: September 27, 2016; accepted: March 24, 2017; available online: March 31, 2017

DOI: $10.17535 /$ crorr.2017.0015

\section{Introduction}

When designing a service center deploying, a responsible designer must comply with randomly occurring events, which may endanger accessibility of the service by individual users [3], [5], [7], [11]. The uncertainty caused by detrimental events can be handled by several ways. The most commonly employed way is a robust system design, where robustness is achieved by taking into account various probable scenarios caused by random detrimental events. In this case, the emergency system is designed so that the resulting deployment of service centers minimizes the maximal objective function value over all considered scenarios [2], [10], [12], [14]. The scenarios for an emergency system design are usually described by particular matrices of time-distances from a possible center location to a user location.

${ }^{\dagger}$ Corresponding author 
In this way, each possible detrimental event corresponds to an evaluated consequence in the form of extended travel times.

The usual way of taking into account all scenarios [2], [14] is based on minimizing the maximal objective function of the individual instances corresponding to particular scenarios. The min-max link-up constraints represent a computational burden due to bad convergence of the branch-and-bound method embedded inside most available IP-solvers.

To overcome this drawback, we suggest an iterative algorithm, which processes much smaller problems, and in which the uncertainty given by scenarios is replaced by fuzziness of individual time-distances. In other words, we assume that the time-distances are fuzzy values in the suggested approach. The optimization process commonly used for solving mathematical programming problems with fuzzy coefficients follows the Tanako-Asai's iterative approach [15], where the level of satisfaction is subsequently increased so that the associated constraints are not broken.

The goal of this contribution is to introduce the above mentioned iterative algorithm and to provide a comparison of the suggested approach to the standard approach [2] from the point of computational time and robustness. Based on the associated result analysis, we will answer the question as to whether the two approaches are substitutable.

The presented paper is organized as follows. The second section comprises radial formulation of the robust emergency system design together with a summarization of the solution methods studied in [10]. The third section focuses on the fuzzy approach to the emergency system design, where also radial formulation of the problem is used. The forth section reports on the results of numerical experiments performed by the two approaches and yields a brief comparative analysis of the designed service center deployments. The obtained findings are summarized in the fifth section.

\section{Robust emergency system design using scenario set}

The robust emergency system design problem with radial formulation [1], [4], [6], [8] can be described according to [9], [10]. We employ the following denotations. Let symbols $J$ and $I$ denote the set of user locations and the set of possible service center locations, respectively. Symbol $b_{j}$ denotes the number of users, who share the location $j$, and $p$ denotes the maximal number of service centers, which can be chosen from $I$. The objective is to minimize the maximal system disutility over the set $U$ of scenarios by a suitable deployment of the $p$ centers. We distinguish scenario $b \in U$, which is the basic scenario corresponding to the standard conditions. The system disutility under a given scenario is defined as the sum of all disutility values associated with the individual users. The value of a user's disutility is given by the mutual positions of the user location and the location of 
the service center providing the service. We assume that the user's disutility grows with increasing distance between the user and the service center. Disutility following from the distance between locations $i$ and $j$ under a specific scenario $u \in U$ is denoted here as $d_{i j u}$. The values of $d_{i j u}$ may be proportional to the network distances between the users' location $j$ and the center location $i$ under the scenario $u$. Our assumption is that the relevant disutility of a user may range over a limited domain starting at zero and ending at some maximal value. The considered domain can be approximated by a range of integers. In this paper, we consider that each value of $d_{i j u}$ is an integer and less than or equal to the maximal value $D_{\max }$ from the domain. The decisions, which determine the designed public service system, can be modeled by introduced decision variables $y_{i} \in\{0,1\}, i \in I$. The variable takes the value of 1 if a service center is located at $i$ and it takes the value of 0 otherwise. The variable $h$ denotes the upper bound of the system disutility over the set $U$ of individual scenarios.

Let us define $v=D_{\max }-1$. Further, auxiliary zero-one variables $x_{j s u}$ for $s=0 \ldots v$ and $u \in U$ are introduced to complete the radial model. The variable $x_{j s u}$ takes the value of 1 , if the disutility of the user at $j \in J$ from the nearest located center under the scenario $u \in U$ is greater than $s$ and it takes the value of 0 otherwise. Then the expression $x_{j 0 u}+x_{j 1 u}+\ldots+x_{j v u}$ constitutes the value of disutility $d_{j u^{*}}$ from user location $j$ to the nearest located service center under scenario $u \in U$. Let us introduce a zero-one constant $a_{i j u} s$ under scenario $u \in U$ for each triple $[i, j, s]$, where $i \in I, j \in J, s \in[0 . . v]$. The constant $a_{i j u}{ }^{s}$ is equal to 1 , if the disutility $d_{i j u}$ between the user location $j$ and the possible center location $i$ is less than or equal to $s$, otherwise $a_{i j u}{ }^{s}$ is equal to 0 . Then the radial-type min-sum robust emergency service system design problem according to [2] can be formulated as model (1)(7), where the maximum of the objective function values over the set $U$ of scenarios is minimized. We introduce auxiliary decision variable $h$ to model the upper bound of the mentioned maximum.

\section{Minimize $\quad h$}

Subject to: $\quad x_{j s u}+\sum_{i \in I} a_{i j u}^{s} y_{i} \geq 1 \quad$ for $j \in J, \quad s=0,1, \ldots, v, u \in U$

$$
\sum_{i \in I} y_{i} \leq p
$$

$$
\sum_{j \in J} b_{j} \sum_{s=0}^{v} x_{j s u} \leq h \quad \text { for } u \in U
$$

$$
y_{i} \in\{0,1\} \text { for } i \in I
$$




$$
\begin{gathered}
x_{j s u} \geq 0 \text { for } j \in J, s=0,1, \ldots, v, u \in U \\
h \geq 0
\end{gathered}
$$

In this model, the objective function (1) represented by variable $h$ gives the upper bound of the all objective function values over the set $U$ of individual scenarios. The constraints (2) ensure that the variables $x_{j s u}$ are allowed to take the value of 0 , if at least one center is located in radius $s$ from the user location $j$ and constraint (3) limits the number of located service centers by $p$. The link-up constraints (4) ensure that each perceived disutility is less than or equal to the upper bound $h$. As concerns the obligatory constraints (6), only values zero and one are expected in any feasible solution, but it can be seen that the model has an integrality property restricted to the variables $x_{j s u}$. It follows that the relevant values of $x_{j s u}$ in the optimal solution will be equal to one or zero without imposing binary constraints upon these variables.

As the min-max link-up constraints (4) represent an undesirable burden in any integer programming problem due to bad convergence of the branch-and-bound method, we explored the possibility provided by Lagrangean relaxation to overcome this burden [10]. The Lagrangean relaxation was applied on the constraints (4) only. Each of these constraints was associated with a non-negative Lagrangean multiplier $\lambda_{u}$ and sub-gradient method was used to set up suitable values of the multipliers.

\section{Robust emergency system design using fuzzy values}

The fuzzy approach does not deal with the set of given crisp scenarios, but it describes the individual uncertain values, e.g. the value of perceived disutility, by a range of possible values together with a measure of relevance of the individual values from the range. The measure is called membership function and the function is defined on the whole set of real numbers and maps this definition range on the interval of real numbers from zero to one. The value of one is assigned to the real values which belong to the fuzzy value at the highest level and the value of zero is intended for real values outside the range of the fuzzy value. A particular fuzzy set defined on real numbers where the membership function satisfies the given conditions [7], [13], [15] is called the fuzzy number. In this paper, we restrict ourselves to a special type of the fuzzy sets intended for expressing that a processed uncertain disutility value is greater than or equal to a given real value $d_{i j-M a x}$ with tolerance $d_{i j-M a x}-d_{i j-m i n}$.

Having described the uncertain disutility following from the time-distance between locations $i$ and $j$ by fuzzy set $\boldsymbol{d}_{i j}$ with the membership function $\mu_{d i j}$ depicted in Fig. 1, we can define function $\underline{d}_{i j}(t)$ as a function giving the lowest 
disutility value which belongs in the range $\left[d_{i j-m i n}, d_{i j-M a x}\right]$ of the fuzzy set $\boldsymbol{d}_{i j}$ at the level of satisfaction $t$.

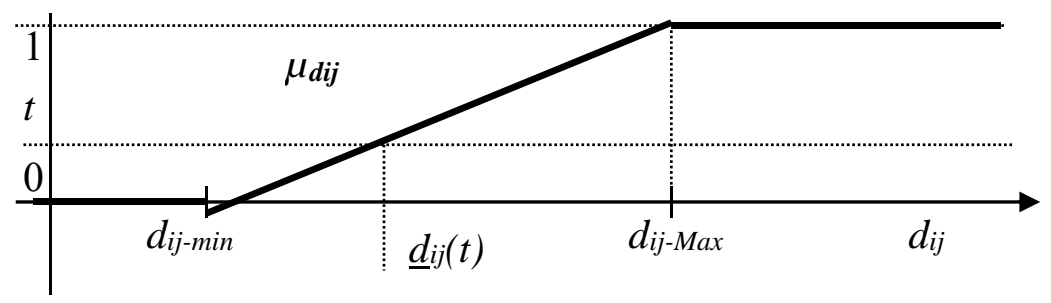

Figure 1: The membership function of a fuzzy set $\boldsymbol{d}_{i j}$.

Then we can define a zero-one constant $a_{i j}{ }^{s}(t)$ for each pair $[i, j]$ and the given level of satisfaction $t$, where $i \in I, j \in J, s \in[0 . . v]$ and $t \in[0,1]$. The constant $a_{i j}{ }^{s}(t)$ is equal to 1 , if the disutility $\underline{d}_{i j}(t)$ between the user location $j$ and the possible center location $i$ is less than or equal to $s$, otherwise $a_{i j}{ }^{s}(t)$ is equal to 0 .

After these preliminaries, we can formulate the emergency system design problem at the level of satisfaction $t$ using the location variables $y_{i}$ defined in the previous section and auxiliary variables $x_{j s}$ for $s=0 \ldots v$, which are redefined in the following way. The variable $x_{j s}$ takes the value of 1 , if the disutility $\underline{d}_{i j}(t)$ of the user at $j \in J$ from the nearest located center is greater than $s$, otherwise it takes the value of 0 . Then the expression $x_{j 0}+x_{j 1}+x_{j 2}+\ldots+x_{j v}$ constitutes the value of disutility $d_{j^{*}}$ from user location $j$ to the nearest located service center at the level of satisfaction $t$.

The fuzzy approach to a general minimization mathematical programming problem consists in determining the highest level of satisfaction $t$, for which the associated constraints are satisfied and the objective function value belongs to a fuzzy set of satisfactorily small values of the objective function. The fuzzy set of satisfactorily small values is usually constructed from two real values $F^{1}$ and $F^{2}$, where $F^{1}$ corresponds to the optimal objective function value for the most favorite case of the problem coefficients, and $F^{2}$ corresponds to the optimal objective function value for the least favorite case of the problem coefficients. The membership function $\mu_{\text {Usmall }}(F)$ is shown in Fig. 2.

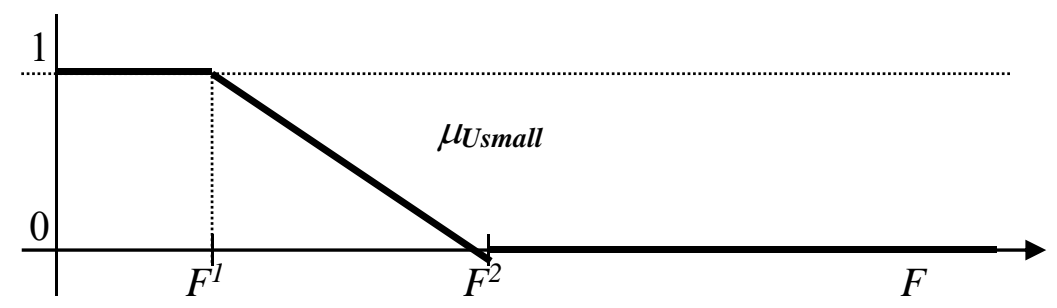

Figure 2: The membership function of a fuzzy set of sufficiently small values. 
The constraint ensuring that the objective function value $F$ belongs to the sufficeently small values of system disutility at a level of satisfaction $t$ follows.

$$
F=\sum_{j \in J} b_{j} \sum_{s=0}^{v} x_{j s} \leq t F^{1}+(1-t) F^{2}
$$

Now, we can complete the model with the following constraints.

$$
\begin{gathered}
x_{j s}+\sum_{i \in I} a_{i j}^{s}(t) y_{i} \geq 1 \quad \text { for } j \in J, \quad s=0,1, \ldots, v \\
\sum_{i \in I} y_{i} \leq p \\
y_{i} \in\{0,1\} \text { for } i \in I \\
x_{j s} \geq 0 \quad \text { for } j \in J, \quad s=0,1, \ldots, v
\end{gathered}
$$

Next, we formulate the problem as follows.

\section{Maximize $t$ \\ Subject to: (8)-(12)}

The objective of the problem is the maximization of the level of satisfaction $t$ subject to constraints (8)-(12). Due to constraint (9), the problem is nonlinear and difficult to solve. That is why, we use an iterative approach, which is known as the Tanako-Asai's method used in the fuzzy optimization [15].

The method searches for a feasible solution to the problem formulated for a fixed value of $t$. If a feasible solution is found, the value of $t$ is then increased, the associated model of the problem is reformulated and the searching process is repeated. In the opposite case, when no feasible solution exists, the next examined value of $t$ is a little less. Upon a subsequent search for feasible solutions for the increased or decreased values of $t$, the optimal value can be estimated with an arbitrary precision $\varepsilon$. Let us denote the solving procedure for the problem defined by the linear program minimize $F$ subject to (8)-(12) for a fixed value of $t$ as $\operatorname{GetOpt}(t)$. Let $(\boldsymbol{y}, \boldsymbol{x})$ denote the resulting solution. We can then implement the Tanaka-Asai's method in accordance to the following steps, where $\varepsilon$ is the demanded precision of the maximal level of satisfaction.

0 . Set $t_{\min }=0, t_{\max }=1$.

1. If $t_{\max }-t_{\min } \geq \varepsilon$, go to step 2, otherwise terminate.

2 . Set $t=\left(t_{\max }+t_{\min }\right) / 2$ and go to step 3 .

3. Apply procedure GetOpt(t) and go to step 4 . 
4. If no solution $(\boldsymbol{y}, \boldsymbol{x})$ exists, set $t_{\max }=t$, otherwise set $t_{\min }=t$ and update the best found solution $(\boldsymbol{y}, \boldsymbol{x})^{\text {best }}$ and $t^{\text {best }}$. Go to step 1 .

\section{Numerical experiments}

In this section, we present an overview of numerical experiments aimed at ascertaining the characteristics of suggested approaches based on scenarios and fuzzy sets. The comparison was performed in terms of computational time and the resulting emergency service system design.

To solve the problems described in the previous sections, the optimization software FICO Xpress 8.0 (64-bit, release 2016) was used and the experiments were run on a $\mathrm{PC}$ equipped with the Intel ${ }^{\circledR} \mathrm{Core}^{\mathrm{TM}}$ i7 $5500 \mathrm{U}$ processor with the parameters: $2.4 \mathrm{GHz}$ and $16 \mathrm{~GB}$ RAM.

The used benchmarks were derived from a real emergency health care system, which was originally implemented in selected regions of Slovakia. For each selfgoverning region, i.e., Bratislava (BA), Banská Bystrica (BB), Košice (KE), Nitra (NR), Trenčín (TN), Trnava (TT) and Žilina (ZA), all cities and villages with the corresponding number of inhabitants $b_{j}$ were used. The coefficients $b_{j}$ were rounded to hundreds. These sub-systems cover demands of all communities towns and villages spread over the particular regions by a given number of ambulance vehicles. In the benchmarks, the set of communities represents both the set $J$ of user locations and also the set $I$ of possible service center locations. The cardinalities of these sets vary from 87 to 515 according to the considered region. The number $p$ of located centers was derived from the original design and it varies from 9 to 52 . To enrich the set of benchmarks, we added also several instances for the self-governing region of Žilina, which were solved for different values of parameter $p$. The network distance from a user to the nearest located service center was taken as the user's disutility. The achieved results are summarized in the Table 1.

An individual experiment was organized so that the referential system design was suggested for the basic scenario $b$ only without taking into account the other scenarios. The particular results for basic scenario are reported in the "BASIC" part of the table. The computational time in seconds is denoted by "СT" and the objective function value of a resulting solution $\boldsymbol{y}$ for basic scenario $b$ is computed according to (13). We should note that the ObjF is computed for resulting solutions of basic, standard and fuzzy approaches and the corresponding values are denoted by ObjFB, ObjFS and ObjFF respectively.

$$
\mathrm{ObjF}=\sum_{j \in J} b_{j} \min \left\{d_{i j b}: i \in I, y_{i}=1\right\}
$$


To compare the objective functions of the robust designs (min-max objective function) of a resulting solution $\boldsymbol{y}$, we use the expression (14). We should note that the ObjFM is computed for the resulting solutions of standard and fuzzy approaches and the corresponding values are denoted by ObjFMS and ObjFMF respectively.

$$
\operatorname{ObjFM}=\max \left\{\sum_{j \in J} b_{j} \min \left\{d_{i j u}: i \in I, y_{i}=1\right\}: u \in U\right\}
$$

After the referential system design corresponding to the usual situation on the transportation network was obtained, the robust design using the model (1) - (7) was computed taking into account all studied scenarios. Due to the lack of common benchmarks for studying robustness, the scenarios used in our computational study were created in the following way. We selected 25 percent of the matrix rows with the rows corresponding to the biggest cities in regard to the number of system users. Then we chose randomly from 5 to 15 rows from the selected ones and the associated disutility values in the chosen rows were multiplied by the randomly chosen constant from the range 2, 3 and 4. The rows, which were not chosen by this random process, remain unchanged (they are multiplied by the value of 1 ). In this way, 10 different scenarios were generated for each self-governing region. The scenarios represent the consequence of fatal detrimental events, when some time-distances are several times elongated. The achieved results for the robust emergency service system design are reported in the middle part of the table. As before, "CT" denotes the computational time in seconds. The column "ObjFS" contains the objective function value computed according to (13) for the basic scenario and values of location variables $y_{i}$ resulting from the solution of the model (1)-(7). To evaluate the price paid for making the system resistant to various catastrophic events, the price of robustness "PoR" was computed. Its value is defined as the difference between "ObjFS" and "ObjFB" expressed as a percentage of "ObjFB". Both mentioned values "ObjFS" and "ObjFB" are computed for the basic scenario and the particular resulting values of location variables $y_{i}$. The right part of the table is used for the results of fuzzy approach explained in Section 3. The objective function value "ObjFF" was evaluated using the expression (13) for the basic scenario and the values of location variables $y_{i}$ resulting from the solution of the model (8)-(12). The column "Dif" is used to report the difference between the objective function values "ObjFF" and "ObjFS" expressed as a percentage of "ObjFB" computed for the basic scenario. The negative values of "Dif" indicate such cases, in which the fuzzy approach gave a better solution in regard to the objective function value (13). The standard robust and fuzzy designs can be compared using the Hamming distance "HD", which is defined as follows. Let $\boldsymbol{y}^{r}$ denote the vector of location 
variables for the standard robust design and let $\boldsymbol{y}^{f}$ denote the vector for the fuzzy one. Then, the Hamming distance "HD" takes the form of (14).

$$
H D=\sum_{i \in \hat{I}}\left(y_{i}^{r}-y_{i}^{f}\right)^{2}
$$

Hamming distance evaluates the structural difference between two designs in the sense that it informs of the number of locations in which the designs differ, but does not refer to the quality of the designs. Therefore, we also compared the robust and fuzzy design from the point of the other characteristics mentioned above. As shown in Table 1, the emergency service system designs obtained using the standard robust model (1)-(7) and suggested fuzzy approach do not differ as much. From the point of computational time, the fuzzy approach seems to be more suitable when middle-sized instances are solved. Some higher values of "PoR" can be explained by the fact that the robust design takes into account all scenarios and possible failures, whereas the basic design concerns only the basic scenario.

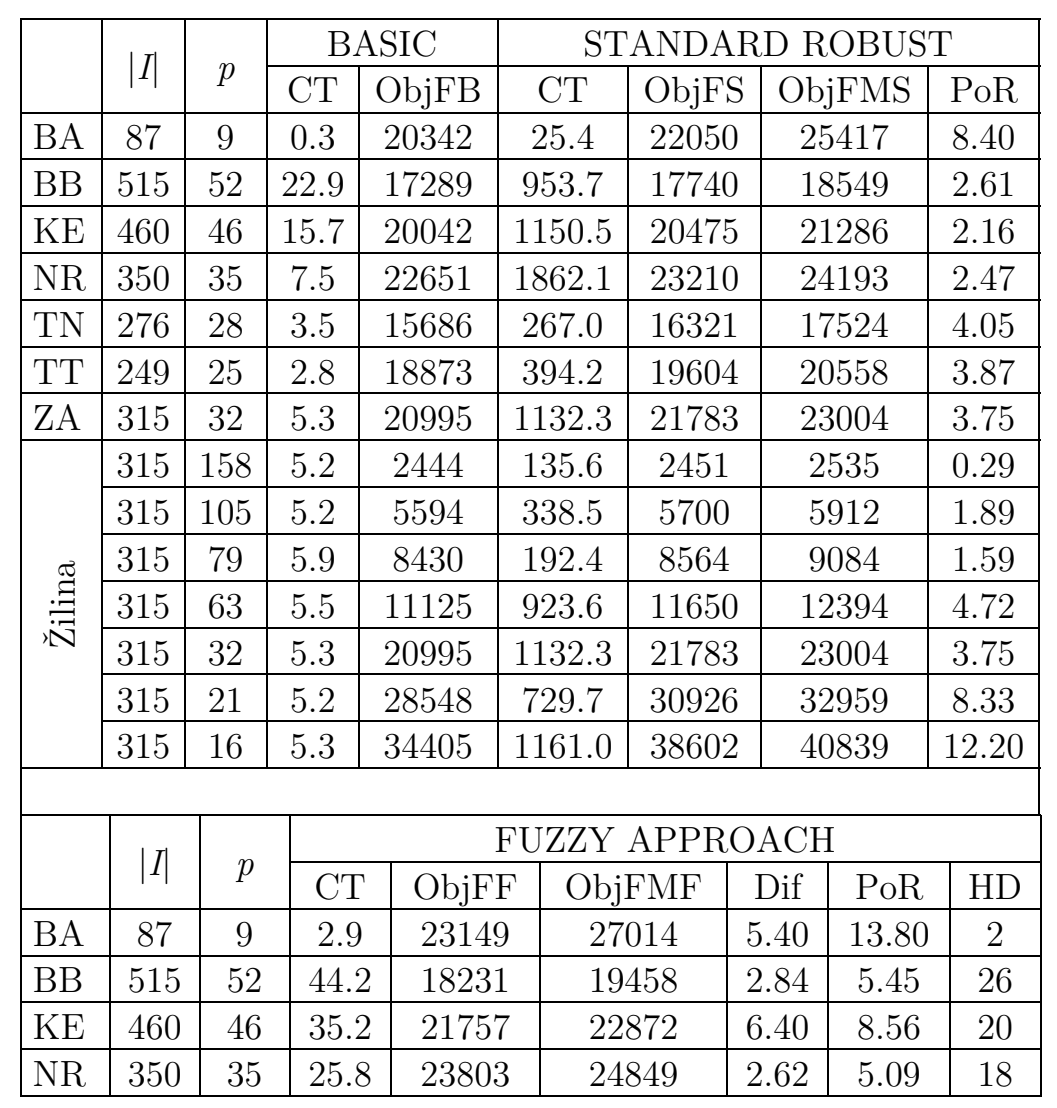




\begin{tabular}{|c|c|c|c|c|c|c|c|c|}
\hline $\mathrm{TN}$ & 276 & 28 & 16.0 & 16173 & 17883 & -0.94 & 3.10 & 8 \\
\hline $\mathrm{TT}$ & 249 & 25 & 10.2 & 20603 & 21639 & 5.29 & 9.17 & 14 \\
\hline $\mathrm{ZA}$ & 315 & 32 & 13.9 & 22391 & 23655 & 2.90 & 6.65 & 14 \\
\hline \multirow{7}{*}{ 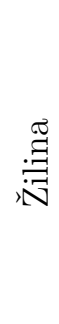 } & 315 & 158 & 13.5 & 2525 & 2623 & 3.03 & 3.31 & 26 \\
\hline & 315 & 105 & 13.9 & 5789 & 6331 & 1.59 & 3.49 & 16 \\
\hline & 315 & 79 & 14.0 & 9021 & 9994 & 5.42 & 7.01 & 20 \\
\hline & 315 & 63 & 14.1 & 12476 & 13525 & 7.42 & 12.14 & 30 \\
\hline & 315 & 32 & 13.9 & 22391 & 23655 & 2.90 & 6.65 & 14 \\
\hline & 315 & 21 & 16.6 & 31260 & 34420 & 1.17 & 9.50 & 8 \\
\hline & 315 & 16 & 14.5 & 37450 & 41432 & -3.35 & 8.85 & 14 \\
\hline
\end{tabular}

Table 1: The results of numerical experiments for the self-governing regions of Slovakia

\section{Conclusions}

This paper has focused on mastering real-sized instances of the emergency service system design problem under uncertainty using a commercial IP-solver. The uncertainty follows from the requirement of making the designed system resistant to various catastrophic events, which may occur in the transportation network, through which the associated service is provided. The uncertainty can be processed in different ways. In this paper, we have compared the robust approach based on scenarios with the fuzzy approach, where the disutility values are modelled using fuzzy numbers. The achieved results have confirmed that the fuzzy approach gives $\mathrm{r}$ results much faster and it is acceptable also in terms of solution accuracy. Our conclusion based on the performed computational study is that we have presented a useful tool for the robust emergency service system design problem, which can be easily implemented using common commercial optimiza-tion software.

Future research in this field could focus on finding relevant scenarios, which may significantly impact the performance of an emergency service system. Since a robust design can significantly differ from the basic one, it would be useful to find a method that allows changing only a limited number of centers in comparison with the standard solution.

\section{Acknowledgement}

This work was supported by the research grants VEGA 1/0518/15 "Resilient rescue systems with uncertain accessibility of service", VEGA 1/0463/16 "Economically efficient charging infrastructure deployment for electric vehicles in smart cities and communities", APVV-15-0179 "Reliability of emergency systems on infrastructure with uncertain functionality of critical elements". 


\section{References}

[1] Avella, P., Sassano, A. and Vasil'ev, I. (2007). Computational study of large scale p-median problems. Mathematical Programming, 109, pp. 89-114

[2] Correia, I., Saldanha da Gama, F. (2015). Facility locations under uncertainty. Location Science, eds. Laporte, Nikel, Saldanha da Gama, pp. 177-203

[3] Current, J., Daskin, M. and Schilling, D. (2002). Discrete network location models. Facility location. Applications and theory, eds. Drezner Z. et al. Berlin: Springer, pp. 81-118

[4] García, S., Labbé, M., Marín, A. (2011). Solving large p-median problems with a radius formulation. INFORMS Journal on Computing 23 (4), pp. 546556

[5] Ingolfsson, A., Budge, S., Erkut, E. (2008). Optimal ambulance location with random delays and travel times. Health Care management science, 11 (3), pp. 262-274

[6] Janáček, J. (2008). Approximate covering models of location problems. In: Lecture Notes in Management Science: Proceedings of the 1st International Conference ICAOR '08, Vol. 1, Sept. 2008, Yerevan, Armenia, ISSN 20080050, pp. 53-61

[7] Janáček, J. (2015). Public service system design with fuzzy parameters of perceived utility. In Central European Journal of Operations Research, 23 (3), pp. 595-606

[8] Janáček, J., Kvet, M. (2011). Approximate solving of large p-median problems. In: ORP3-Operational research peripatetic post-graduate programme: proceedings, Cádiz, Spain, September 13-17, 2011, Cádiz: Servicio de Publicaciones de la Universidad de Cádiz, 2011, pp. 221-225

[9] Janáček, J., Kvet, M. (2013). Public service system design with disutility relevance estimation. In Proceedings of the 31st international conference "Mathematical Methods in Economics", September 11-13, 2013, Jihlava, Czech Republic, pp. $332-337$

[10] Janáček, J. and Kvet, M. (2016). Designing a robust emergency service system by Lagrangean relaxation. In Mathematical Methods in Economics 2016, Liberec, Czech Republic, September 6th-9th, 2016, pp. 349-353

[11] Marianov, V. and Serra, D. (2002). Location problems in the public sector. In Drezner, Z. (Ed.). Facility location - Applications and theory, Berlin: Springer, pp. 119-150

[12] Pan, Y., Du, Y. and Wei, Z. (2014). Reliable facility system design subject to edge failures. American Journal of Operations Research, 4, pp. 164-172

[13] Ramík, J., Vlach, M. (2002). Generalized concavity in fuzzy optimization and decision analysis. Kluwer Academic Publishers, Boston, 296 p.

[14] Scaparra, M.P., Church, R.L.: Location problems under disaster events. In: Location Science, eds. Laporte, Nikel,Saldanha da Gama, pp. 623-642 
[15] Teodorovič, D., Vukadinovič, K. (1998). Traffic control and transport planning: A fuzzy sets and neural networks approach. Boston: Kluwer Academic Publishers, $387 \mathrm{p}$. 